\title{
AUTOPERCEPCIÓN DE LAS RECLUSAS REINCIDENTES EN UNA CÁRCEL COLOMBIANA Y SU RELACIÓN CON EL CRIMEN
}

\author{
CARMELINA PABA BARBOSA ${ }^{1}$ / YESID JOSÉ ORTEGA PACHECO ${ }^{2}$ / MAIRA \\ ALEJANDRA CAMPILLO GÓMEZ ${ }^{3}$ / MICHAEL FABIÁN HERNÁNDEZ \\ BOLIVIVR $^{4}$ / MILENA MARTÍNEZ RUDAS ${ }^{5}$ / DANIELA MARCELA PORTO \\ CORREDOR $^{6}$
}

\section{RESUMEN}

Con el objetivo de conocer por primera vez cuál es la autopercepción de las reclusas reincidentes en una cárcel colombiana y su relación con el crimen, se realizó metodológicamente un estudio de naturaleza cualitativa con un diseño fenomenológico. La información se obtuvo de entrevistas semiestructuradas aplicadas a la población de estudio y codificada a través del software Atlas.ti., los datos arrojados sustentan su validez gracias a la técnica triangulación de la información donde intervienen estos últimos, lo arrojado a la luz de la teoría y lo hallado por los investigadores. Los resultados muestran que las convictas no se auto conciben como delincuentes y que esta no tiene relación directa con los actos delincuenciales.

Palabras clave: Autopercepción, Reclusas, Crimen, Delincuencia.

\footnotetext{
${ }^{1}$ Doctora en Psicología con énfasis en Neurociencias Cognitivas Aplicadas. Directora del Programa de Psicología, Universidad del Magdalena (Colombia). Directora del Grupo de Investigación Cognición y Educación, Universidad del Magdalena, Santa Marta (Colombia). Carrera 32, no. 22-08. Código Postal 470004, Santa Marta, Colombia. Correo electrónico: carmelinapaba@gmail.com

${ }^{2}$ Psicólogo de la Universidad del Magdalena (Colombia). Asociado al Grupo de Investigación Cognición y Educación, Universidad del Magdalena, Santa Marta (Colombia). Carrera 32, no. 22-08. Código Postal 470004, Santa Marta, Colombia. Correo electrónico: yesid.1594@gmail.com

${ }^{3}$ Psicóloga de la Universidad del Magdalena (Colombia). Asociada al Grupo de Investigación Cognición y Educación, Universidad del Magdalena, Santa Marta (Colombia). Carrera 32, no. 22-08. Código Postal 470004, Santa Marta, Colombia. Correo electrónico: malejaquintero88@gmail.com

${ }^{4}$ Psicólogo de la Universidad del Magdalena (Colombia). Asociado al Grupo de Investigación Cognición y Educación, Universidad del Magdalena, Santa Marta (Colombia). Carrera 32, no. 22-08. Código Postal 470004, Santa Marta, Colombia. Correo electrónico: michaelhdez19@gmail.com

${ }^{5}$ Magister en Psicología Jurídica de la Universidad de la Costa, (Colombia). Doctorante en Psicología Jurídica y Forense en la Universidad de Santiago de Compostela (España). Coordinadora del Área de Psicología Jurídica de la Universidad de la Costa, Calle 58, no. 55-66. Código Postal 080002, Barranquilla, Colombia. Correo electrónico: milenamartinezrudas@gmail.com

${ }^{6}$ Psicóloga de la Universidad del Magdalena (Colombia). Asociada al Grupo de Investigación Cognición y Educación, Universidad del Magdalena, Santa Marta (Colombia). Carrera 32, no. 22-08. Código Postal 470004, Santa Marta, Colombia. Correo electrónico: danyporto12@gmail.com
} 


\section{SELF-PERCEPTION OF THE RECENT RECLUENCES IN A COLOMBIAN} JAIL AND THEIR RELATIONSHIP WITH THE CRIMER

\section{ABSTRAC}

In order to know first what is the perception of the recidivist inmates in a Colombian jail and its relationship to crime, a qualitative study with a phenomenological design was methodologically conducted. The information was obtained from semi-structured interviews applied to the study population and codified through Atlas.ti software. The data support its validity thrown through technical triangulation of information which involved the latter, the light thrown theory and found by researchers. The results show that convicted not self-conceived as criminals and that this has no direct relationship with criminal acts.

Keywords: Self-perception, Prisoners, Crime, Delinquency.

\section{Introducción}

A nivel mundial, los problemas sobre delincuencia han aumentado significativamente afectando cada día más a la población y, por ende, a las sociedades, tal y como lo explican Dammert y Arias (2007) y Téllez (2015), este es uno de los principales conflictos que acogen a Latinoamérica, por tal motivo, se ubica como el segundo continente a nivel mundial más violento en el mundo. Según las estadísticas de la Oficina de las Naciones Unidas contra la Droga y el Delito (UNODC) en Latinoamérica, los principales delitos hallados son: homicidio, con un registro aproximado de 144.733 personas en 2011, delitos tradicionales y delitos relacionados con drogas, la mayoría de ellos efectuados por hombres, y en un bajo porcentaje se evidencia la participación de la mujer en estos actos punibles. Durante el 2006 y 2010 la población carcelaria femenina de América Latina creció, pasando de 40,000 a más de 74,000 internas, reflejando un aumento en la población penitenciaria de este tipo (Oficina de las Naciones Unidas contra la Droga y el Delito (UNODC, 2010). 
En América Latina y el caribe, las mujeres delincuentes encarceladas representan el $12 \%$ de la población en general, cifra que se ha mantenido en la región en los últimos años según los estudios realizados por Carranza en el año 2012. Además, según el informe de la Secretaría General de Instituciones Penitenciarias en ese mismo año, desde el último mes del año 2011, la tasa de personas en cárceles era de 70.472 internos y solo la mujer representaba el 7,5\% (Fontanil, Alcedo, Fernández y Ezama, 2013). De ello, en los centros penitenciarios a nivel mundial se enmarcan dentro del $5 \%$ de toda la población reclusa en general (Giacomello, 2013).

En Colombia, las tasas de delincuencia han crecido de manera desmedida, resaltando las diferentes problemáticas de orden social, económico y político que contribuyen de una u otra manera a que esto se mantenga. Subsiguientemente, el país habría registrado por primera vez luego de transcurrido aproximadamente siete años un incremento significativo en el porcentaje semestral de homicidios en contraste con la primera mitad del año 2012 a la del 2013, pasando de 6.918 a 7.380 de los casos respectivamente (El Universal, 2013). De acuerdo a un estudio realizado por la Universidad Sergio Arboleda (2013), las cifras de homicidio en términos semestrales estuvieron enmarcadas en 34,4\%. Para el año 2014 el registro aproximado fue de 671.234 (Buitrago, Rodríguez y Bernal, 2015).

La participación de la mujer en la criminalidad ha sido uno de los temas olvidados en los que el estado colombiano ha invertido poco para conocer, prevenir y ejecutar políticas públicas lo suficientemente eficaces para contrarrestar este fenómeno (Norza-Céspedes, González-Rojas, Moscoso-Rojas y González-Ramírez, 2012), tal como se puede evidenciar a continuación.

Según el INPEC (2014), la población femenina estuvo compuesta por 8.977 reclusas de las cuales 6.200 fueron condenadas por los diferentes crímenes cometidos. Con relación a los índices de criminalidad femenil en enero de 2016, la cifra de mujeres en las cárceles del país estuvo enmarcada en 8.240 , representando así el 6,8\% de la población carcelaria y penitenciaria total (INPEC, 2016), lo que permite observar el gran número de 
delincuentes femeninas en los últimos cinco años. Por lo anterior, se hace necesaria la implementación de estrategias eficaces por parte del gobierno a favor de disminuir considerablemente el número no solo de mujeres sino de hombres en los centros de reclusión y además, brindarles todas las herramientas necesarias para evitar su reincidencia.

Los estudios tanto nacionales como internacionales sólo han tenido como objetivo demostrar las causas y consecuencias de la conducta delictiva por parte de las mujeres pero han olvidado factores como los sentimientos, las motivaciones, su autocritica y la opinión. Al trasladarse a la ciudad de interés, Valledupar, se observa que la tasa de delincuencia durante el año 2013 fue aproximadamente de 5.369 delitos ejecutados por ambos géneros, con un promedio diario de 15 por día, ubicándose a nivel nacional con una participación total del 0.83\% (Duarte, 2013). Además, las acciones criminales con relación al hurto en la cadena de suministros (piratería terrestre) se incrementó un $25 \%$ en todo el país y en esta se registraron 5 respectivamente.

En el 2014, se han capturado en flagrancia 624 personas y 94 por orden judicial (Barco y Arana, 2014). Sin dejar de lado el incremento que ha tenido la criminalidad juvenil, registrándose capturas a más de 170 jóvenes en el año 2013 y en el 2014 se reconocieron aproximadamente 203 casos de delito infantojuvenil (El Informador, 2014). Actualmente, los centros reclusorios cuentan con 1.412 presos de los cuales 453 son mujeres (INPEC, 2016). Al mismo tiempo, no se puede desligar el factor pobreza, ya que según algunos autores, esta puede estar fuertemente ligado a la delincuencia cuando se habla sobre el tema. Falta de oportunidades, poco nivel de estudios o, no tener un empleo, son solo algunas de las variantes que sobresalen en personas que han cometido delitos tales como hurtos, homicidios y venta de estupefacientes (Pérez y Pinzón, 2009), siendo vistos como única salida para el sustento de los mismos y sus familias, convirtiéndose así en una de las mayores problemáticas que acoge al país (Mertz, 2010). 
Desde este punto de vista, resulta importante comprender el proceso de autopercepción de las reclusas reincidentes en una cárcel Judicial colombiana y su relación con el crimen. Ello con el objetivo de, generar a futuro estrategias que contribuyan a disminuir las conductas criminales, conocer sus características y cómo articulan su autopercepción con el delito, saber quiénes son, el porqué de sus actos que van en contra de la ley, para qué, cómo se perciben así mismas y si existe o no relación directa entre la conducta delictiva y el auto concepto.

Además de esto, se contribuirá por una parte a las ciencias criminológicas y sus sub áreas entendiendo esta como aquella disciplina científica e interdisciplinaria la cual tiene como objetivo el estudio y análisis del delito, el criminal y la criminalidad en lo que se enmarca la víctima, la pena por parte del delincuente y la repercusión social del acto delictivo. Además de identificar las causas, trabajar en ellas y evitarlas (Fuentes, 2008).

También, resulta relevante socialmente debido a que da a conocer más allá de las cifras, las tasas o los índices, una mayor compresión de la realidad que viven las mujeres en prisión, en lo que se enmarcan factores como los sentimientos, las motivaciones y la autocrítica. Contribuye, por una parte, a cambiar la percepción que tiene la sociedad sobre dicha población y que, antes de juzgar como es el común denominador frente una situación socialmente no aceptada, se logre entender sin justificar el porqué de dicho acto, logrando así un mayor entendimiento. Ello, debido a que en un gran número de procesos, este se ve influido por factores criminógenos, definiéndose como "aquellos elementos que en conjunto favorecen a un determinado resultado antisocial" (Hikal, 2005, p. 45). Por otra, este trabajo busca contribuir a los diferentes organismos del distrito como la alcaldía, la defensoría del pueblo, la policía de la ciudad, la Fiscalía, la Sijín y el Instituto Colombiano de Bienestar Familiar (ICBF) para el accionar de las diferentes estrategias que puedan asistir dicha problemática desde sus primeras etapas. 
Por último, la realización de este estudio permite instruirse respecto a los fenómenos de delincuencia desde otra mirada diferente al derecho o la legislación constitucional, resaltando además que esta es la primera investigación de este tipo que se realiza en el país y que dicho estudio puede servir como apoyo para otras investigaciones encaminadas en esta línea y contribuir al desarrollo de modelos o teorías explicativas de las conductas criminales.

Pero ¿qué tienen que decir las mujeres reincidentes que gracias a las conductas punibles cometidas están en condición de reclusas en una prisión o cómo se perciben ellas luego de haber cometido dichos actos?, ¿culpables o inocentes?, ¿existirá alguna relación entre la autopercepción y el delito? o ¿simplemente víctimas de condiciones poco favorables con las que cuenta el país para el desarrollo de sus ciudadanos?

\section{Estado del arte}

\subsection{Marco conceptual}

Según Martínez (2011), el proceso de autopercepción está relacionado con la capacidad que tiene un sujeto de describirse a sí mismo, de conocer cuál es su autoconcepto y de dar juicios de valor sobre él. Por consiguiente, es importante resaltar las tres fases que comprende la autopercepción, la cual está relacionada con la concepción que posea el sujeto: 1. Autoidentidad, se entiende como "la autopercepción de sí mismo, a partir de una configuración básica de características físicosociales y psicosociales, asociadas a las subculturas de base y a las categorías sociales o grupos de pertenencia, con los cuales se identifica el actor social" (p.11); 2. Autoconcepto, refiere a "las percepciones del individuo sobre sí mismo, las cuales se basan en sus experiencias con los demás y en las atribuciones que él mismo hace de su propia conducta" (Shavelson, Hubner y Stanton, 1976, citado por Cazalla-Luna y Molero, 2013, p. 2); y 3. Autovaloración corresponde a la "evaluación y valoración del Mí del actor social (y de Yo, marginalmente, salvo excepción), y en consecuencia de sus conductas más permanentes". El actor social se autoevalúa y es evaluado 
por "otros", y en base a estas evaluaciones, se autovalora (Cooper, 2002, citado por Sandoval, 2007, p. 124).

Por otra parte, el término reclusa hace referencia a aquella persona de género femenino que se encuentre privada de la libertad por haber cometido un delito. Comprendiendo que para la conducta criminal existen factores criminógenos que precipitan a que esta se realice, siendo estos "elementos que favorecen un determinado resultado antisocial y que a su vez están constituidos por factores endógenos y exógenos que influyen en el desarrollo anormal de una persona, y que éstos pueden llevar a la realización de ciertas conductas antisociales" (Hikal, 2005, p. 45). Y reincidencia "se refiere a la repetición de un acto delictivo por parte del delincuente" (Ossa, 2012, p. 115).

\subsection{Antecedentes investigativos}

De acuerdo con un estudio realizado por Giraldo, Ocampo, Tuta, Gallego y López en el 2015, las causas de la conducta criminal en la ciudad de Cali (Colombia) está comprendida de la siguiente manera: pobreza y la exclusión social: el 19,1\% de la población vive en condiciones vulnerables de pobreza y son estigmatizados socialmente como delincuentes, familias disfuncionales, cultura de la ilegalidad e incursión y práctica de la ilegalidad. Se puede concluir que las conductas delincuenciales en la ciudad se pueden generar y promover hacia otras ciudades del país afectando no solo a la población general colombiana, sino también la imagen que posee la ciudad tanto a nivel nacional como internacional.

En este sentido, Morillas, Patró y Aguilar (2011) citados por Aguilar (2012), plantean la presencia de factores de riesgo determinantes que se relacionarían con la predisposición hacia el crimen y una incapacidad para auto percibirse como delincuentes, tales como: "impulsividad o comportamientos arriesgados, actitudes $\mathrm{O}$ pensamientos negativos, problemas de control de ira y trastornos mentales o de comportamiento", los cuales son determinantes a la hora de ejecutar actos delictivos.

De igual forma, un estudio realizado por Sandoval (2007), encontró que un gran porcentaje de jóvenes delincuentes cuya experiencia en delitos 
era constante, poseían una autopercepción distinta a lo que se espera que posean de acuerdo con su rol de criminales, es decir, estos se autopercibían como criminales cuando comenzaron a realizar conductas punibles, cuando asimilan que esto se convierte en su forma de vivir. "Cuando roban frecuentemente comienzan a ser reconocidos y respetados por sus pares, siendo etiquetados como delincuentes, tanto por ellos mismos, como por el entorno y cuando el robar, es definido por ellos como un trabajo" (Sandoval, 2007 , p. 227). Consecuentemente, se halló que algunos jóvenes reconocían su error y planificaban acciones con miras a cambiar su rótulo de criminales, pero no en todos los casos, ya que, aludían que muchas de las acciones criminales fueron producto de errores cometidos en su vida.

Del mismo modo, se tuvo en cuenta la visión y sentido de la reincidencia en la mayoría de ellos que manifestaron presentar la voluntad de volver a delinquir, sin embargo, dicho concepto cambia de acuerdo al hecho que le implicaría volver a estar privados de la libertad.

Con relación, algunas investigaciones han logrado justificar la relación bidireccional entre el modo en que los reclusos se perciben a sí mismos y la forma en como suelen interpretar el mundo. Luego de esto, se permite establecer el grado de culpabilidad o inocencia en el crimen, ya que, la personalidad del sujeto se transforma en un factor concluyente a la hora del reconocimiento de sus actos, es decir, el individuo examina sus apreciaciones cognitivas y motivacionales que lo conllevaron a cometer el delito. De acuerdo con Garrido, Montoro y López (1992) y Ross (1992) citado por López (2006), a partir de allí se puede interpretar aspectos de la personalidad del sujeto que caracterizan su conducta delictiva, es decir que es necesario el análisis y estudio de las esferas cognitivo-motivacionales, teniendo en cuenta que demuestra "cómo percibe y se representa un sujeto determinada situación, cómo la soluciona, qué juicios hace sobre la misma o qué proceso determina su elección delictiva" (López, 2006, p. 82).

Por otra parte, Aguilar (2012) esboza el término de Autoconsciencia Personal del Riesgo (APR), la cual hace mención sobre el propio conocimiento del accionar criminal por parte del delincuente donde los 
comportamientos antisociales son aceptados por el mismo. En este sentido, se relaciona con la capacidad que posee un individuo de reconocer el alcance de sus actos y ser consciente de los mismos con el fin de generar acciones a raíz de las consecuencias producidas que le permitan un cambio a nivel cognitivo y comportamental. Por lo tanto, aquellos reclusos que han sido presos en algún centro penitenciario durante un periodo de su vida y no desarrollan la capacidad de conciencia sobre el daño provocado para generar un cambio, se caracterizan generalmente por ser personas que están en contacto permanente con factores de riesgos predisponentes al delito y la criminalidad. Teniendo en cuenta que dichos comportamientos suelen aparecer en edades tempranas como la niñez y que se refuerzan en la adolescencia y la adultez.

Según un estudio realizado por Arenas y Luna (2012) el cual tuvo como objetivo central realizar una caracterización psicológica en este tipo de población, determinó que la crianza que recibieron las reclusas reincidentes por parte de sus progenitores sirvió como factor determinante a la hora de cometer los actos punibles de manera reiterada, ya que son estos los que proporciona un estilo de vida determinado que estas asumen, volviéndose un patrón a seguir. Además, afirman que "la mujer reincidente se caracteriza por ser impulsiva, narcisista, evitar vínculos con figuras de autoridad, son aisladas, poseen dificultad para establecer relaciones interpersonales y con actitud de desconfianza, cautela excesiva y con gran tendencia de interpretar los estímulos inadecuados".

\section{Método}

\subsection{Tipo de estudio}

El presente estudio se rige bajo el paradigma de investigación cualitativa, el cual estudia las situaciones tal cual, y como se presentan, tratando de dar sentido a todos aquellos fenómenos que resultan ser significativos para los sujetos que entran en contacto con este (Castillo, Jaimes, y Chaparro, 2001). 


\subsection{Diseño}

El enfoque de investigación es fenomenológico. Este se enfoca en "explicar los significados en los que estamos inmersos en nuestra vida cotidiana, y no las relaciones estadísticas a partir de una serie de variables, el predominio de tales o cuales opiniones sociales, o la frecuencia de algunos comportamientos" (Rodríguez, Gill y García, 1997).

\subsection{Participantes}

La población de estudio del presente proyecto investigativo fueron diez (10) reclusas reincidentes en una cárcel judicial colombiana. De ello, la muestra estuvo compuesta por siete (7) reclusas condenadas las cuales fueron seleccionadas a través del muestreo no probabilístico por conveniencia, en la que los participantes que hacen parte de la investigación son seleccionados a criterio del investigador, seleccionando a los sujetos fácilmente accesibles (Salkind, 1998).

\subsection{Instrumentos}

La técnica de recolección de información implementada para el desarrollo del presente proyecto fue la entrevista semiestructurada. Esta se encarga de recoger todos los datos proporcionados por los individuos que participan en la investigación por medio de un conjunto de preguntas formuladas de manera abierta, las cuales tiene un orden estipulado, es decir un protocolo o guion previamente elaborado. Cabe resaltar, que al momento de la implementación de la misma pueden surgir nuevas preguntas que favorezcan los fines científicos del trabajo siendo de gran utilidad al momento de analizar los datos (Mayan, 2001).

\subsection{Análisis de datos}

La interpretación de la información recogida a través de las participantes del presente proyecto se realizó gracias al análisis de contenido; técnica utilizada para "describir de forma objetiva, sistemática y analítica la información adquirida por diversos medios como la entrevista" (Varguillas, 2006, p. 75) y cuyo proceso de análisis se realizó gracias al software ATLAS.ti, herramienta utilizada para la comprensión de los datos 
suministrados por distintas técnicas de recolección de información, ya que, permite codificar, categorizar y estructurar los hallazgos más significativos (Varguillas, 2006).

De igual manera se tuvieron en cuenta las consideraciones éticas pertinentes para esta clase de estudios. El proceso de acceso al campo se llevó a cabo durante 2 etapas diferentes: la primera llamada "Inicio", consistió en la identificación de la población a trabajar, la selección de la muestra y el proceso elaboración de la entrevista semiestructurada, y la segunda conocida como "cierre", comprendió el análisis de contenido, el análisis de la información, presentación de resultados, conclusiones y la socialización del trabajo investigativo.

\section{Resultados}

Dando cumplimento a la etapa de cierre, en la que se analizó el contenido de las entrevistas, se procedió a la realización de las categorías, subcategorías y códigos atendiendo a los lineamientos planteados en el proyecto investigativo. Se utilizó el software ATLAS.ti que permitió una mayor comprensión de la información como se presenta a continuación. En la siguiente tabla se muestran de manera resumida los resultados obtenidos y que fueron organizados mediante la aplicación del análisis de contenido.

Cuadro 1. Presentación resultados codificados

\begin{tabular}{|c|c|c|}
\hline CATEGORIAS & SUBCATEGORIAS & CÓdIGOS \\
\hline \multirow{3}{*}{ AUTOPERCEPCIÓN } & Factores internos & Autoconcepto \\
\hline & Cambios & Negativos \\
\hline & Relaciones familiares & $\begin{array}{l}\text { Familia formada } \\
\text { Familia de origen }\end{array}$ \\
\hline \multirow[t]{2}{*}{ CRIMEN } & Motivos & $\begin{array}{l}\text { Personales } \\
\text { Económicos }\end{array}$ \\
\hline & Antecedentes & Familiares \\
\hline
\end{tabular}

Fuente: Elaboración propia con base en el discurso de las participantes del estudio. 
Con base al discurso de las reclusas reincidentes se establecieron dos grandes categorías, la primera llamada Autopercepción y la segunda conocida como Crimen, cada una con su respectiva subcategorías y códigos. En relación a la categoría de Autopercepción, esta se compone de la subcategoría de Factores Internos, Cambios y Relaciones Familiares. La primera de ellas está compuesta por el código de Autoconcepto, del cual las entrevistadas expresaron:

"...buena persona, cariñosa y amable" (Sujeto 3).

"Me percibo bien, como buena" (Sujeto 2).

"Yo soy una persona buena" (Sujeto 4).

"Pues... de buen corazón, muy noble, tengo que cambiar muchas cosas" (Sujeto5),

"Soy bien, una buena mujer" (Sujeto 6).

En cuanto a la segunda subcategoría compuesta por el código Negativos, las participantes señalan:

"Ninguno..." (Sujeto 1).

"No sé, ninguno, creo..." (Sujeto 2).

"Pues yo creo que nada" (Sujeto 3).

"Pues engordar, y me siento como aplacada... ninguno la verdad" (Sujeto 6).

"Ninguno, siento más rencor" (Sujeto 7).

Igualmente, los códigos de Familia Formada y Familia de Origen pertenecen a la subcategoría de Relaciones Familiares en la que la primera de estas hace referencia al funcionamiento de su hogar y la dinámica familiar y el segundo, a la relación en los diferentes ámbitos en lo que las reclusas se desenvolvían con sus pares (padres, hermanos, etc.).

Por consiguiente, para el primer código las convictas aluden:

"Es buena, si buena" (Sujeto 1).

"Bien, si buena" (Sujeto 2).

"Buena relación, pero últimamente me he alejado" (Sujeto 3).

"Buena, me han apoyado gracias a Dios" (Sujeto 4).

"Es espectacular" (Sujeto 6).

Del mismo modo, el discurso que comprende al código de Familia de Origen es el siguiente:

"Buena, todo fue bien..." (Sujeto 1).

"No me quejo, fue muy buena" (Sujeto 2).

"Buena relación con mi madre y mi familia materna, el entorno bien, tuve buena crianza, nada de malos hábitos" (Sujeto 3). 
"Buena, me dieron buena crianza" (Sujeto 4).

"Si, una buena relación" (Sujeto 6).

Por otra parte, la categoría de Crimen está comprendida por las subcategoría de Motivos, la cual hace mención a las razones por las que se cometieron los actos punibles, formada por los códigos Personales y Económicos.

De acuerdo al primero de estos, las reclusas expresaron causas subjetivas en su accionar delictivo, alegando lo siguiente:

"Siempre me ha gustado matar, no se..." (Sujeto 1).

"En mi es normal..." (Sujeto 2).

"No tenía necesidad, porque quise" (Sujeto 3).

Además, la mayoría de las entrevistadas manifestaron que la causa principal por la cual cometieron el crimen era por falta de dinero, alegando que:

"Por la necesidad de plata, me metí en una banda..." (Sujeto 4).

"Por necesidad... no hay plata" (Sujeto 5).

"Para poder comprar, no hay nada... la plata, tu sabes..." (Sujeto 6).

"Por plata... económicos, por eso" (Sujeto 7).

En concordancia con la segunda subcategoría de Antecedentes, que alude al historial delictivo por parte de su familia y los conocimientos que tengan sobre los mismos. El código que lo conforma es Familiares. Durante la entrevista las presas mencionaron:

“...mi mamá por porte y droga, por conseguir plata” (Sujeto 1).

"Si, por asesinatos, la mayoría de mi familia" (Sujeto 2).

"Si, la adicción a la droga, por necesidad, casi toda mi familia" (Sujeto 3).

"Un hermano expendedor, le dieron 10 años por droga" (Sujeto 6).

"Mi mamá expendedora y hermano robo y droga" (Sujeto 7). 


\section{Discusión}

De acuerdo a la categoría de Autopercepción, esta refiere al "concepto que se tiene de uno mismo sobre sus actitudes, deseos y expectativas; el proceso se realiza en el interior de cada individuo" (Martínez, 2011).

Con relación a esto, los reclusos manifestaron lo siguiente: “...buena persona, cariñosa y amable" (Sujeto 3), "Me percibo bien, como buena" (Sujeto 2), "Yo soy una persona buena" (Sujeto 4), "Pues... de buen corazón, muy noble, tengo que cambiar muchas cosas" (Sujeto 5), "Soy bien, una buena mujer" (Sujeto 6). Con relación a lo anterior, Clemmer, (1940); Pinatel, (1969); y Goffman, (1979) citados por Pérez y Redondo (1991) aluden que debido al efecto de prisionización, "concebida en términos de asimilación por los internos de hábitos, usos, costumbres, y cultura de la prisión", las reclusas van construyendo su autodescripción de tal forma que dicho efectos influyen directamente en la concepción que tiene el individuo de sí mismo producto de un proceso de encarcelamiento y sus comportamientos posteriores a recuperar la libertad lo que hace que puedan llegar a percibirse como delincuentes.

En el presente caso se logró evidenciar que para esta población independientemente del entorno carcelario el autoconcepto que tienen las reclusas es de ser personas buenas y de bien, aptas para vivir en sociedad y con buenos valores éticos y morales.

Subsiguientemente, manifiestan no haber tenido ningún cambio positivo dentro de prisión mencionando: "Ninguno..." (Sujeto 1), "No sé, ninguno, creo..." (Sujeto 2), "Pues yo creo que nada" (Sujeto 3), "Pues engordar, y me siento como aplacada... ninguno la verdad" (Sujeto 6). "Ninguno, siento más rencor" (Sujeto 7). Según Dechiara, Furlani, Gutiérrez, y Kratje (2009) plantean que los individuos presentan cambios producto de la experiencia condicionante tanto social como psicológica que vive dentro de prisión. Además, según el efecto de prisionización planteados por Clemmer, (1940); Pinatel, (1969); y Goffman, (1979) citados de Pérez y Redondo (1991), se deben presentar algunos cambios en la conducta delictiva en 
aspectos positivos por la estadía en la cárcel pero según el discurso de las reclusas se halló que estas no lo presentan por lo que manifestaron no tener ninguna transformación en su forma de pensar, concebir la vida, estilos y hábitos de vidas, afirmando ser las mismas de siempre dentro de prisión y fuera de esta al momento de obtener su libertad.

Del mismo modo, Copello (2009), citado por Dechiara et al, (2009) manifiesta que "el individuo deja de tener la posibilidad de continuar con su vida social, familiar y laboral. La añoranza de todo lo perdido se vuelve una constante en el pensamiento del preso" (Dechiara et al, 2009, p.175).

Pero de acuerdo con el discurso de las participantes, se logra observar que ellas declaran que sus relaciones familiares son lo suficientemente buenas y satisfactorias lo cual no es uno de los temas primordiales a tratar dentro de prisión, teniendo en cuenta que mantienen una comunicación estable y sin ningún tipo de problemas. Además, no reprochan nada de la crianza recibida por parte de sus padres afirmando que la enseñanza dejada por estos a lo largo de su desarrollo evolutivo fue lo suficientemente buena y aceptable. Cabe resaltar que no se profundizó en la pregunta, debido a que la institución carcelaria no permitió por motivos internos, la finalidad de las mismas por lo que se denota superficial, obteniendo un resultado somero en el conocimiento en sus respuestas.

Aunque el término crimen no tiene una realidad ontológica (Hillyard y Tombs, 2013), Hikal (2005) lo define como "un acto que implica motivos individuales y antisociales que afectan la integridad social". Las reclusas expresaron tener motivos principalmente económicos los que conllevaron a ser un factor decisivo al momento de delinquir, aludiendo que: "Por la necesidad de plata, me metí en una banda..." (Sujeto 4), "Por necesidad... no hay plata" (Sujeto 5), "Para poder comprar, no hay nada... la plata, tu sabes..." (Sujeto 6), "Por plata... económicos, por eso" (Sujeto 7).

Sin embargo, no se descarta la posibilidad de alguno de los crímenes cometidos tiene un trasfondo como una enfermedad mental que conlleve a dicho acto, tal y como estas lo refieren: "Siempre me ha gustado matar, no 
se..." (Sujeto 1), "En mi es normal..." (Sujeto 2), "No tenía necesidad, porque quise" (Sujeto 3).

Siguiendo con esta línea, Arroyo y Ortega (2009) plantean que "una parte importante de las personas que ingresan en un centro penitenciario, lo hacen debido a un comportamiento socialmente inadaptado. En algunos casos como manifestación de un proceso patológico que les ha llevado a entrar en conflicto con la ley" y por otro, la falta de dinero.

Por consiguiente, se plantea que un gran número de mujeres que se hallan en reclusorios penales tienen distintos motivos justificables por los cuales cometieron el delito, por tal motivo, se logró observar que muchas asumen con resignación el castigo que reciben por sus actos. Igualmente, no se desconoce que alguno de los crímenes cometidos tiene un trasfondo como una enfermedad mental que conlleve a dicho acto, además varios de estos tienen una base en la falta de oportunidades en relación al trabajo, lo cual conlleva a la búsqueda de dinero de manera fácil.

Pérez y Pinzón (2009), con relación a los antecedentes familiares, encontraron que muchas veces el estilo de crianza de los padres determina a futuro el comportamiento de sus hijos. En sus estudios, los sujetos se caracterizaron por tener padres autoritarios, permisivos y un débil establecimiento de reglas de estos hacia sus progenitores determinando a posteriori la conducta delictiva de sus hijos y la influencia de estos en sus actos vandálicos.

Asimismo, "el abandono del hogar a temprana edad por parte de los participantes es producto del conflicto provocado por la confusión o cambio de roles a nivel del hogar cuando no está presente uno de los miembros" (Pérez y Pinzón, 2009). Por lo tanto, en relación a los datos proporcionados por las reclusas se logra evidenciar los diversos factores que pueden llegar a afectar a un individuo desde su infancia y por ende, en un futuro llegar a cometer un delito con la finalidad de sobrevivir ante el medio. De tal modo que desde muy jóvenes se instaura en su pensamiento el actuar de forma criminal, agregándole a esto la existencia de familiares que han estado involucrados en diferentes actos delictivos, por lo cual, muchas veces 
reciben la aprobación de esto por parte de sus familiares, incentivación a continuarlos y aceptación al momento de realizar los crímenes. Además, reafirman los motivos económicos como factor precipitante pata cometer algún tipo de delito.

\section{Conclusiones}

A través de los datos recogidos para estudiar y analizar los aspectos implicados en la autopercepción de las reclusas reincidentes en una cárcel judicial colombiana y su relación con el crimen, se concluye que ellas no se consideran delincuentes a pesar de los actos punibles cometidos que conllevaron a estar reincidiendo en prisión hoy en día. Por otra parte, las entrevistadas manifestaron ser buenas personas, comunes y corrientes que antes de tomar decisiones equivocadas y cometer delitos, llevaban una vida normal como la de cualquier persona común, la diferencia según estas radican en que por cuestiones personales efectuaron las conductas delictivas reiteradamente.

Además, no se evidenció por parte de las convictas sentimientos de arrepentimiento y reconocimiento de culpa por sus actos, por el contrario afirman no haber tenido ningún cambio en su forma de pensar y actuar a lo largo de su vida, teniendo en cuenta las reiteradas ocasiones que fueron capturadas y mucho menos ahora que se encuentran recluidas en prisión cumpliendo la pena impuesta por la ley debido a las conductas tipificadas como delito.

Por otra parte, perciben como funcionales sus ambientes familiares con antecedentes criminales motivados por la falta de dinero y los bajos niveles de estudios por el simple hecho de promover lo que según ellas consideran como buenos valores. Por ello, el factor criminógeno de mayor índole en esta población reclusa es de tipo social, en los que se enmarca la pobreza.

Por tal motivo, se concluye que el proceso de autopercepción no tiene relación directa con el crimen, debido a que la realización de estos actos son productos de las malas decisiones ocurridas en su mayoría por la falta de 
dinero, la escasa educación y la poca empleabilidad resultante de las necesidades que estas debían suplir para poder sobrevivir al medio en el cual se desenvolvían.

\section{Referencias Bibliográficas}

Aguilar, M. M. (2012). La influencia del contexto familiar en el desarrollo de conductas violentas durante la adolescencia: factores de riesgo y de protección, Revista Criminalidad, 54 (2), 27-46.

Arenas, J. A., y Luna, D. C. (2012). Caracterización psicológica de las mujeres privadas de la libertad reincidentes en los delitos de hurto y tráfico, fabricación o portes de estupefacientes en la reclusión de mujeres de Bucaramanga (Tesis de Pregrado). Universidad Pontifica Bolivariana, Medellín, Colombia.

Arroyo, J. M. \& Ortega, E. (2009). Los trastornos de personalidad en reclusos como factor de distorsión del clima social de la prisión, Rev Esp Sanid Penit, 11, (1), 11-15.

Barco, J. N. H. \& Arana, J. E. (2014). Comportamiento de la criminalidad en Colombia, 2013. Revista Criminalidad, 56 (2): 11-33.

Buitrago, J. R., Rodríguez, J. D. \& Bernal, P. A. (2015). Registros administrativos de policía para la consolidación de cifras de criminalidad en Colombia. Revista Criminalidad, 57 (2), 11-22.

Cazalla-Luna, N. y Molero, D. (2013). Revisión teórica sobre el autoconcepto y su importancia en la adolescencia. Revista Electrónica de Investigación y Docencia (REID), 10, 43-64.

Castillo, N. S., Jaimes, G. \& Chaparro R. O. (2001). Una aproximación a la investigación cualitativa. Colombia: Universidad Pedagógica y Tecnológica de Colombia.

Dammert, L. \& Arias, P. (2007). "El desafío de la delincuencia en América Latina: Diagnóstico y respuestas de política". Seguridad y violencia: desafíos para la ciudadanía. FLACSO, 21-66. Recuperado de: http://www.plataformademocratica.org/Publicacoes/124_Cached.pdf

Dechiara, P., Furlani, L. B., Gutiérrez, N. G. \& Kratje, P. (2009). Efectos del cautiverio de las cárceles sobre las personas privadas de libertad, Revista de Epistemología y Ciencias Humanas, 2, 15, 161-190.

Duarte, Y. A. (2013). Resultados operativos de la Policía Nacional, 2012. Revista Criminalidad, 55 (3): 151-156.

El Informador (2014/22 de septiembre). Aumenta la delincuencia juvenil en Valledupar. Recuperado de: http://www.elinformador.com.co/index.php/judiciales/local/85537-aumenta-ladelincuencia-juvenil-en-valledupar 
El Universal. (11/06/2013). Tasas de homicidio habrían vuelto a crecer en Colombia. Recuperado de: http://www.eluniversal.com.co/colombia/tasa-dehomicidios-habria-vuelto-crecer-en-colombia-130877

Fontanil, Y., Alcedo, M. A., Fernández, R. \& Ezama, E (2013). Mujeres en prisión: un estudio sobre la prevalencia del maltrato. RES. Revista Española de Sociología, (20), 21-38.

Fuentes, M. (2008). Criminología: herramienta de la prevención del delito. Del mapa, a la ruta del delito. (Tesis de investigación). Mendoza. Recuperado de: http://www.iusp.uncu.edu.ar/upload/criminologia-basica-para-oficiales-jefes-ysuperiores-policia-de-mendoza-2013.pdf

Giacomello, C. (2013). Mujeres, delitos de drogas y sistemas penitenciarios en América Latina. Consorcio Internacional sobre Políticas de Drogas (IDPCInternational Drug Policy Consortium).

Giraldo, J., Ocampo, J. P., Tuta, F. A., Gallego, M. C. y López, Y. (2015). ¿Por qué las personas ingresan a la delincuencia organizada? Análisis del fenómeno en Santiago de Cali. Revista Criminalidad, 57, (1) 103-119.

Hikal, W. S. (2005). Criminología psicoanalítica, conductual y del desarrollo. México: Flores Editor.

Hillyard, P. \& Tombs, S. (2013). ¿Más allá de la criminología?. Revista Crítica Penal y Poder. (4). 175-196.

INPEC (2014). Informe estadístico enero. Recuperado de: http://www.inpec.gov.co/portal/page/portal/Inpec/Institucion/Estad\%EDsticas/Es tadisticas/Informes \%20y\%20Boletines\%20Estad\%EDsticos/INFORME\%20EST ADISTICO\%20ENEROCORREGIDO03042014.pdf

INPEC (2016). Informe estadístico enero2016. Recuperado de: http://www.inpec.gov.co/portal/page/portal/Inpec/Institucion/Estad\%EDsticas/Es tadisticas/Informes \%20y\%20Boletines\%20Estad\%EDsticos/01\%20INFORME\% 20ENERO\%202016_.pdf

López, M. J. (2006). Psicología de la delincuencia. Ciencias de la seguridad (CISE): Universidad de Salamanca.

Martínez, R. (2011). La influencia de las redes sociales en la autopercepción de los jóvenes que estudian la modalidad Bachillerato Tecnológico. Recuperado

de: http://portal.iteso.mx/portal/page/portal/Dependencias/Rectoria/Dependencias/D ireccion_General_Academica/Dependencias/DESO/Programas_academicos/M C/coloquio_de_proyectos/Coloquio\%20de\%20Proyectos $\% 201 \% 20$ oto\%F10\%20 2011/Propuesta\%20de\%20Investigaci\%F3n\%20ANG\%C9LICA\%20MART\%CD NEZ\%20VF.pdf

Mayan, M. J. (2001). Una introducción a los métodos cualitativos: Modulo de entretenimiento para estudiantes y profesionales. México: Prentice Hall 
Mertz, C. (2010). Delincuencia y otras dimensiones de la pobreza en Chile: resultados preliminares. Recuperado de: http://www.cepal.org/deype/noticias/paginas/8/39508/CMertz_UD.pdf

Norza-Céspedes, E., González-Rojas, A., Moscoso-Rojas, M. \& GonzálezRamírez, J. D. (2012). Descripción de la criminalidad femenina en Colombia: factores de riesgo y motivación criminal. Revista Criminalidad, (54), 1. 339-357. Ossa, M. F. (2012). Aproximaciones conceptuales a la reincidencia penitenciaria, Ratio Juris, 7, (14), 113-140.

Pérez, E. \& Redondo, S. (1991). Efectos psicológicos de la estancia en prisión. Papeles del psicólogo, (48), 11.

Pérez, L. T., Pinzón, X. (2009). Factores psicosociales asociados a la conducta delictiva de los internos condenados por homicidio recluidos en la cárcel de máxima seguridad de Cómbita (Boyacá). Revista Virtual Universidad Católica del Norte, 1 (26).

Rodríguez, G; Gill, J. \& García, E. (1997). Metodología de la investigación cualitativa. España: Aljibe.

Salkind, N. J. (1998). Métodos de investigación. México: Prentice Hall.

Sandoval, C. (2007). "Relatos de vida de jóvenes infractores de ley: una aproximación a sus procesos de reinserción social y comunitaria" (Tesis de Maestría). Universidad de Chile. Santiago de Chile, Chile.

Téllez, W. J. (2015). Explorando la percepción de la delincuencia como principal problema en América Latina. Revista Internacional de Investigación en Ciencias Sociales, 11 (2), 195-208.

Universidad Sergio Arboleda (2013). Sube el homicidio por primera vez en 7 años.

http://www.usergioarboleda.edu.co/Notisergio/homicidio_crece_primer_semestr e_2013.pdf

UNODC. (2010). Situación de la delincuencia y la justicia penal en el mundo. En $12^{\circ}$ Congreso de las Naciones Unidas sobre la Prevención del Delito y Justicia Penal. (pp. 1-2). Salvador, Brasil. Recuperado de: http://www.cinu.mx/XIICongresoONUPrevencionDelito/docs/situacion $\% 20 \mathrm{de} \% 2$ Ola\%20delincuencia.pdf

Varguillas, C. (2006). El uso de atlas.ti y la creatividad del investigador en el análisis cualitativo de contenido upel. Instituto pedagógico rural el mácaro. Revista de educación, 12 (2), 73-87.

\section{Para citar este artículo:}

Paba, C., Ortega, Y., Campillo, M., Hernández, M., Martínez, M. y Porto, D. (2017). Autopercepción de las reclusas reincidentes en una cárcel colombiana y su relación con el crimen. Collectivus, Revista de Ciencias Sociales, 4(1), 6786.

Doi: http://dx.doi.org/10.15648/Coll.1.2017.4 